\title{
A Coarse-Grained Model for Water Involving a Virtual Site
}

\author{
Mingsen Deng, Hujun Shen ${ }^{*}$ \\ Guizhou Provincial Key Laboratory of Computational Nano-material Science, Guizhou Education University \\ No.115, Gaoxin Road, Guiyang, Guizhou, 550018, P. R. China
}

\section{Supporting Information}

\section{Corresponding Author:}

* Dr. Hujun Shen: hujun.shen@hotmail.com 


\section{Analysis of CAVS simulations}

All CAVS simulations under NPT and NVT conditions were performed for $600 \mathrm{~ns}$. For each case, the first $100 \mathrm{~ns}$ run was considered as equilibration run such that the last $500 \mathrm{~ns}$ run was considered as production run for final analysis. Mean value of each physical property was measured as a time average of a single run and the error was estimated as the standard deviation. The NPT simulations were performed to calculate density, electric constant, self-diffusion constant, viscosity, isothermal compressibility, enthalpy of vaporization and hydration free energy, while the NVT simulations were carried out to measure the surface tension of air-liquid interface. To calculate the surface tension, an air-liquid interface was created by increasing the water box size along z-axis after the system was equilibrated under a NPT condition. For instance, the water box size is $6.7 \mathrm{~nm} \times 6.7 \mathrm{~nm} \times 6.7 \mathrm{~nm}$ after the NPT equilibration and is changed to $6.7 \mathrm{~nm} \times 6.7 \mathrm{~nm} \times$ $9.7 \mathrm{~nm}$ in the NVT simulation that is used to evaluate the surface tension.

In this work, to calculate the dielectric constant, the relative dielectric constant and the relative dielectric constant of the reaction field were set to 2.5 and infinity respectively. To compute viscosity $(\eta)$, the following equation is used:

$$
\eta=\frac{k_{B} T}{3 \pi \sqrt{6}} \frac{1}{\sqrt{D^{3} \tau_{s}}}
$$

where $k_{B}$ is the Boltzmann constant, $T$ is the temperature, $D$ corresponds to the self-diffusion constant and $\tau_{s}$ represents the time correlation function (TCF) of the molecular dipole moment. In MD simulations, $D$ and $\tau_{s}$ can be obtained directly using the g_msd and g_dipoles programs implemented in the GROMACS suite.

To measure the hydration free energy of water, one CAVS water is considered as the solute and other CAVS waters are considered as the solvent. One should note that, to distinguish from the CAVS water solvent (CGW), the CAVS water as the solute should be given a different name (such as CGS). The hydration free energy was calculated by using the thermodynamic integration (TI) method ${ }^{1}$ in which the free energy difference $\left(\Delta \mathrm{G}_{\mathrm{hydr}}\right)$ between two states is described by a function of a coupling parameter $(\lambda)$. In this work, 21 NPT simulations (each run has a length of $30 \mathrm{~ns}$ ) were performed at evenly-spaced $\lambda$ spacing from 0 and 1. 
Figure S1. Comparison of Mapping Schemes used in three different CG models: (A) CAVS, (B) MARTINI-POL, and (C) BMW

(A)

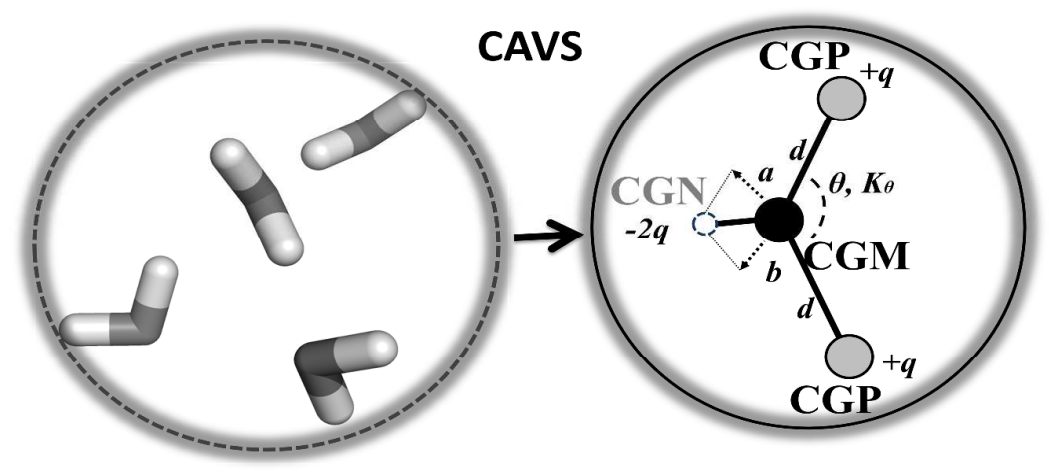

(B)

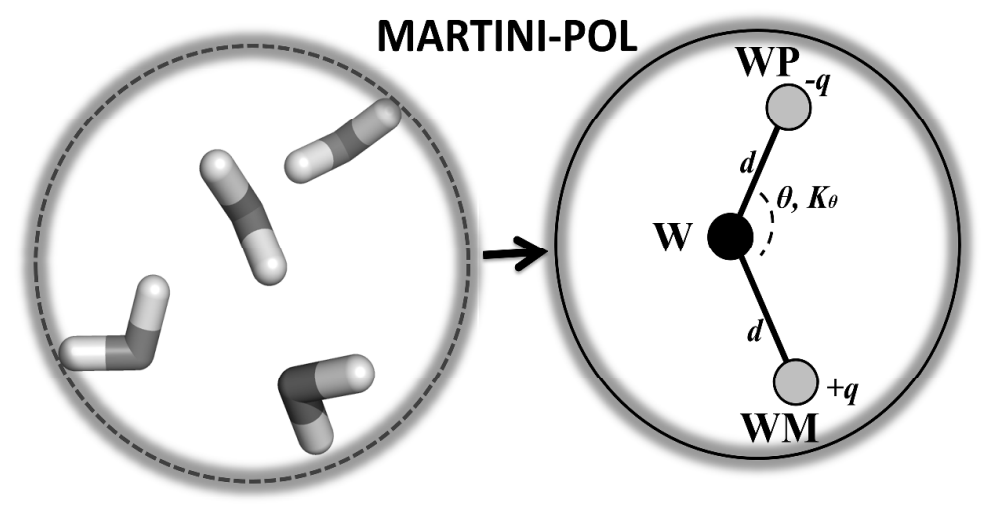

(C)

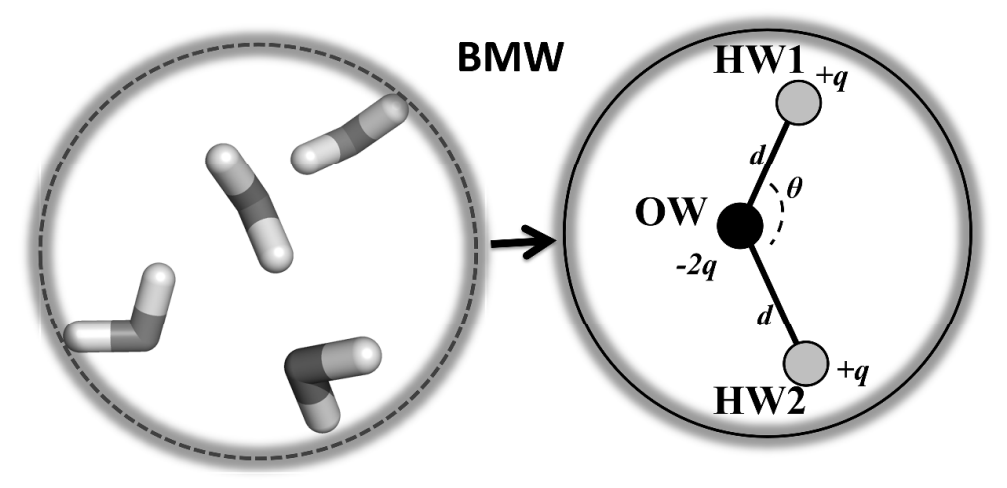


Figure S2. Distributions of the dipole moment magnitude in CAVS water and TIP3P water. The dipole moment for atomistic four-water clusters (by grouping the closest four water molecules) was calculated from a 100-ns NPT simulation of 2000 TIP3P water molecules.

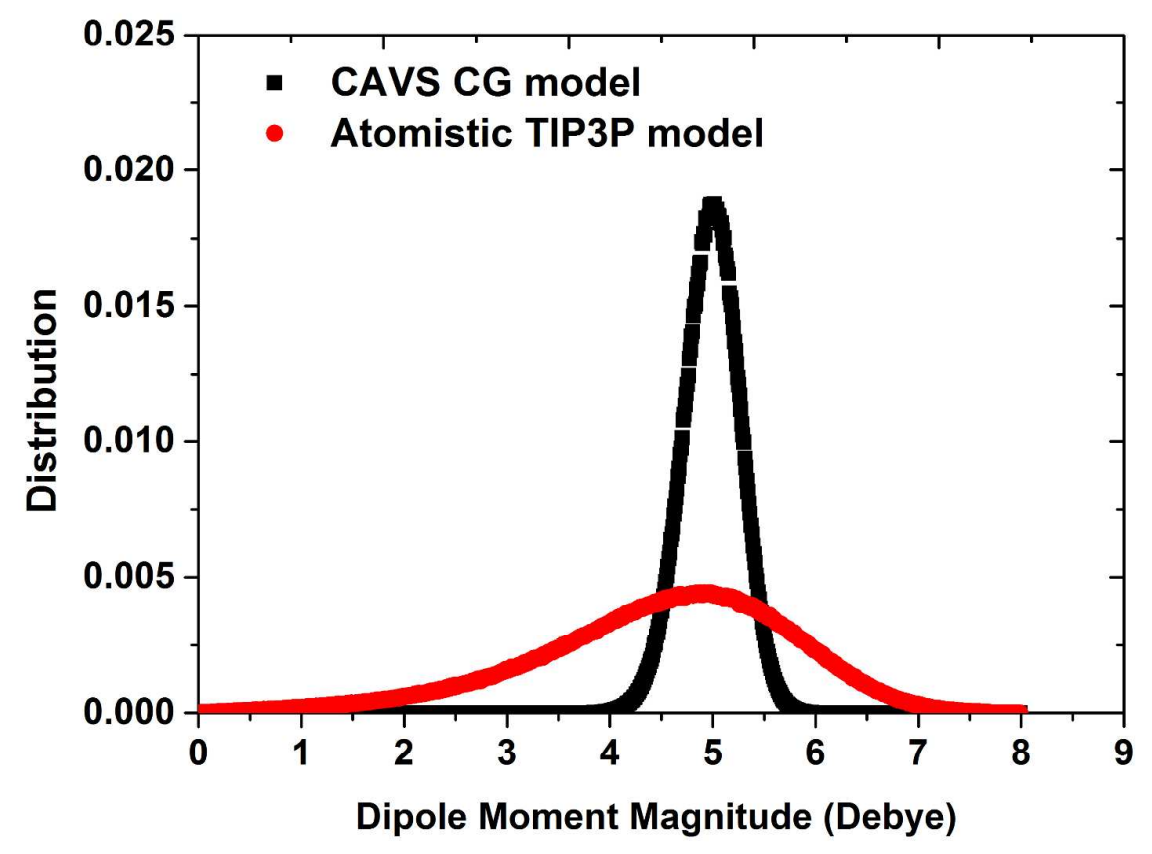


Table S1. Average dipole moment $(\mu)$ and three components $\left(\mathrm{Q}_{\mathrm{xx}}, \mathrm{Q}_{\mathrm{yy}}\right.$ and $\left.\mathrm{Q}_{\mathrm{zz}}\right)$ of quadrupole moment tensors in different $\mathrm{CG}$ models as compared to atomistic model of 4-water clusters.

\begin{tabular}{ccccc}
\hline Models & $\boldsymbol{\mu}$ (Debye) & \multicolumn{3}{c}{ Quadrupole Moment Tensors (Debye $\cdot \AA$ ) } \\
\cline { 3 - 5 } & & $\mathrm{Q}_{\mathrm{xx}}$ & $\mathrm{Q}_{\mathrm{yy}}$ & $\mathrm{Q}_{\mathrm{zz}}$ \\
\hline CAVS & 4.8 & 7.2 & -6.1 & -0.8 \\
BMW & 6.0 & 9.0 & -7.0 & -1.8 \\
MARTINI-POL & 4.9 & 0.0 & 0.0 & 0.0 \\
$\begin{array}{c}\text { Atomistic 4-water } \\
\text { clusters }\end{array}$ & $4.4^{2}, 6.0^{3}$ & 9.0 & -7.2 & -2.0 \\
\hline
\end{tabular}


Table S2 Comparison of physical properties in different water models.

\begin{tabular}{cccccc}
\hline & CAVS & BMW $^{3}$ & MARTINI-POL $^{2}$ & TIP3P $^{4}$ & Experiment \\
\hline Density $\left(\mathrm{kg} / \mathrm{m}^{3}\right)$ & 992 & 1047 & 1043 & 1002 & 997 \\
Dielectric Constant & 76 & 74 & 76 & 82 & 77 \\
Surface Tension & 55 & 77 & 31 & 55 & 73 \\
$(\mathrm{mN} / \mathrm{m})$ & & & & \\
\hline
\end{tabular}


Table S3. Force field Parameters of NaCl for CAVS CG simulations

\begin{tabular}{cccc}
\hline Atoms & Well Depth $\varepsilon(\mathrm{kJ} / \mathrm{mol})$ & Range Parameter $\sigma(\mathrm{nm})$ & Charge $q(\mathrm{e})$ \\
\hline $\mathrm{Na}^{+}$ & 0.3 & 0.25 & 1.0 \\
$\mathrm{Cl}^{-}$ & 0.3 & 0.44 & -1.0 \\
\hline
\end{tabular}




\section{References}

(1) Villa, A.; Mark, A. E. Calculation of the Free Energy of Solvation for Neutral Analogs of Amino Acid Side Chains. J. Comput. Chem. 2002, 23, 548-553.

(2) Yesylevskyy, S. O.; Schäfer, L. V.; Sengupta, D.; Marrink, S. J. Polarizable Water Model for the Coarse-Grained MARTINI Force Field. PLoS Comput. Biol. 2010, 6, e1000810.

(3) Wu, Z.; Cui, Q.; Yethiraj, A. A New Coarse-Grained Model for Water: The Importance of Electrostatic Interactions. J. Phys. Chem. B 2010, 114, 10524-10529.

(4) Jorgensen, W. L.; Jenson, C. J. Temperature Dependence of TIP3P, SPC, and TIP4P Water from NPT Monte Carlo Simulations: Seeking Temperatures of Maximum Density. J. Comput. Chem. 1998, 19, 1179-1186. 


\section{CAVS_CGW.itp}

;,;,;,;; CAVS Coarse-Grained Water

[ moleculetype ]

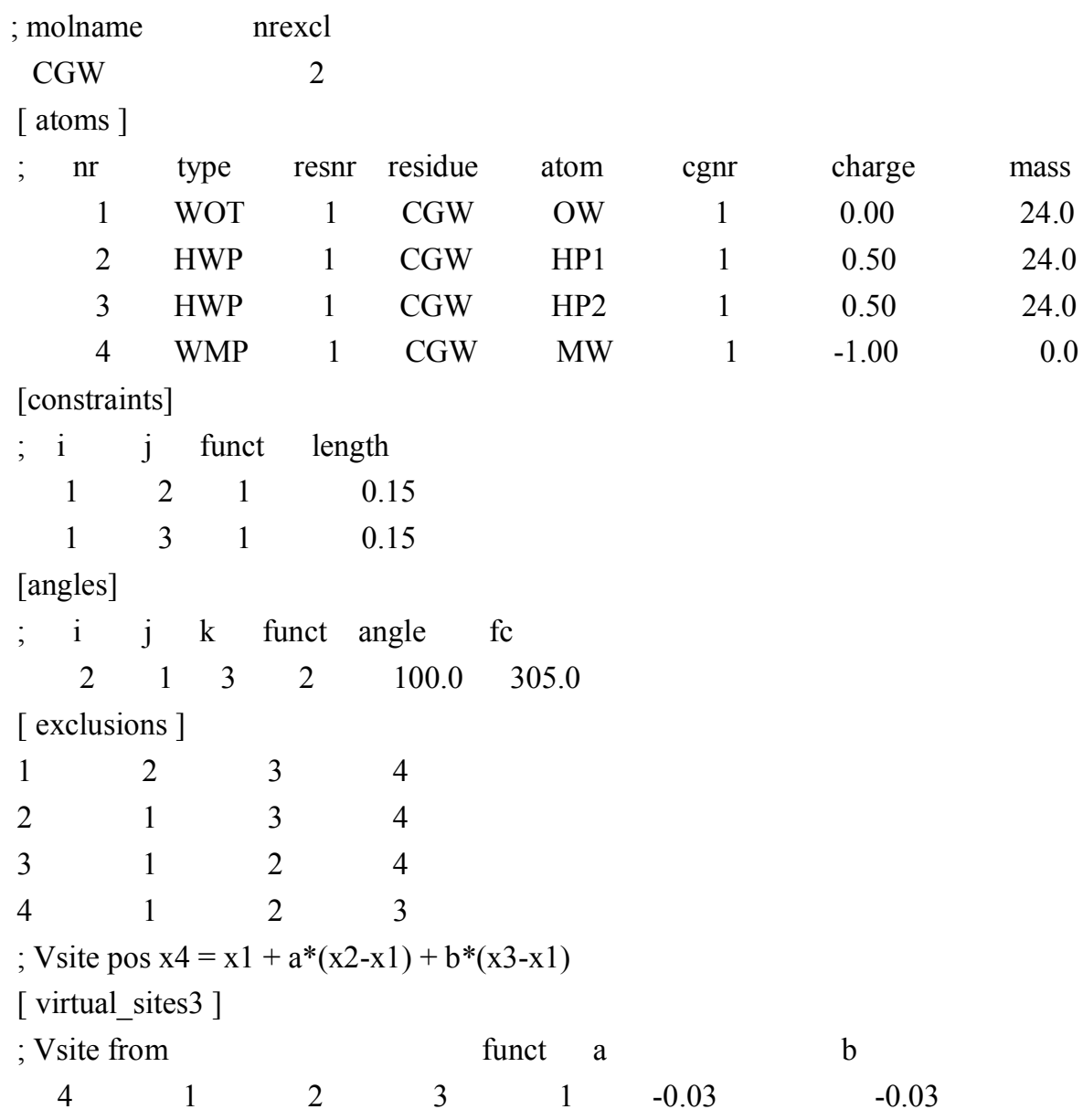

